\title{
GRACE and TIMI risk scores but not stress imaging predict long-term cardiovascular follow-up in patients with chest pain after a rule-out protocol
}

\author{
P. M. van der Zee • H. J. Verberne • J. H. Cornel • \\ O. Kamp • F. M. van der Zant • R. Bholasingh • \\ R. J. De Winter
}

Published online: 17 May 2011

(C) The Author(s) 2011. This article is published with open access at Springerlink.com

\begin{abstract}
Objective To determine the long-term prognostic value of stress imaging and clinical risk scoring for cardiovascular mortality in chest pain patients after ruling out acute coronary syndrome (ACS).

Methods A standard rule-out protocol was performed in emergency room patients with a normal or non-diagnostic admission electrocardiogram (ECG) within $6 \mathrm{~h}$ of chest
\end{abstract}

P. M. van der Zee $\cdot$ R. J. De Winter

Department of Cardiology, Academic Medical Center Amsterdam, Amsterdam, the Netherlands

\section{H. J. Verberne}

Department of Nuclear Medicine,

Academic Medical Center Amsterdam,

Amsterdam, the Netherlands

J. H. Cornel

Department of Cardiology, Medical Center Alkmaar,

Alkmaar, the Netherlands

\section{O. Kamp}

Department of Cardiology, VU Medical Center,

Amsterdam, the Netherlands

F. M. van der Zant

Department of Nuclear Medicine, Medical Center Alkmaar,

Alkmaar, the Netherlands

R. Bholasingh

Department of Cardiology, Slotervaart Hospital Amsterdam,

Amsterdam, the Netherlands

P. M. van der Zee $(\bowtie)$

Department of Cardiology, Academic Medical Center,

room B2-223, Meibergdreef 9,

1105 AZ Amsterdam, the Netherlands

e-mail: p.m.vanderzee@amc.uva.nl pain onset. ACS patients were identified by troponin T, recurrent angina and serial ECG. Dobutamine stress echocardiography (DSE) was performed after ACS was ruled out. Myocardial perfusion scintigraphy (MPS) was performed within 6 months in an outpatient setting according to the physician's discretion.

Results 524 patients were included. GRACE and TIMI risk scores were 75 (57-96) and 1 (0-2) in the rule-out ACS group, and 89 (74-107) and $2(1-3)$ in the ACS group, respectively (median, interquartile range). Follow-up (median 9.4 (8.910.0) years) was complete in $96 \% .350$ of 379 rule-out ACS patients had an interpretable DSE and 52 patients underwent an MPS. 21 of the rule-out ACS patients (6\%) died of a cardiovascular cause compared with 24 (17\%) ACS patients $(p<0.001)$. For rule-out ACS patients, C-statistics were 0.829 and 0.803 for the GRACE and TIMI scores. In these patients, DSE and MPS outcome did not predict long-term cardiovascular mortality. In multivariate analysis, known chronic heart failure, ACE inhibitor use, and GRACE score were independent predictors of cardiovascular mortality.

Conclusions TIMI and GRACE score but not DSE and MPS are accurate predictors of long-term cardiovascular mortality, even in chest pain patients with a normal or non-diagnostic electrocardiogram undergoing a rule-out protocol.

Keywords Chest pain · Echocardiography - Stress imaging · Cardiovascular mortality · GRACE risk score · TIMI risk score

\section{Introduction}

Accurate risk stratification is vital in patients with complaints suggestive of myocardial ischaemia [1,2]. Although 
ECG and cardiac biomarkers are low-cost tools for identification of high-risk patients in need of intensive medical treatment, these tests are hampered by lack of sensitivity and are therefore insufficient for identifying all patients at high risk [3]. Exercise testing is widely performed for diagnostic and prognostic information, but its additional value is limited [4], and the testing is unfeasible in patients with an uninterpretable ECG and in patients unable to exercise $[5,6]$. Stress imaging, such as myocardial perfusion scintigraphy (MPS) or dobutamine stress echocardiography (DSE), may provide an alternative. Available studies on prediction models in acute chest pain patients have a limited follow-up [7-10]. Although reports on the long-term prognostic value of MPS and DSE are extensive, they are limited by the selection of primarily stable patients [11-15]. In a previous prospective study, we reported an important and independent short-term prognostic value of DSE in acute chest pain patients with a normal or non-diagnostic ECG and negative cardiac necrosis markers [16]. The present study investigates the long-term prognostic value DSE and MPS in these patients, in comparison to available clinical risk scores.

\section{Methods}

We conducted a prospective, multicentre study in patients presenting at the emergency room with chest pain as described earlier [16]. Patients who presented at the emergency room within $6 \mathrm{~h}$ after onset of chest pain and who had a normal or non-diagnostic ECG were eligible for inclusion. Excluded were patients younger than 18 years, patients who were unable to give informed consent, and patients with any of the following conditions: atrial fibrillation, conduction disturbances (second or third degree atrial-ventricular block or new bundle branch block), severe uncontrolled hypertension (>180/120 mmHg) despite adequate therapy, severe heart failure, cardiomyopathy, status after resuscitation, serious non-cardiac disease (e.g. infection and neoplastic conditions), and pregnancy. The study was performed in accordance with the principles set out in the declaration of Helsinki and was approved by local ethics committees on human research. Patients remained under observation until at least $12 \mathrm{~h}$ after the onset of symptoms. Evaluation included careful history taking, physical examination, serial 12-lead ECGs, continuous rhythm monitoring, and serial cardiac troponin $\mathrm{T}$ measurements. Serial ECGs were recorded according to protocol directly on admission, after the administration of sublingual nitrates, and during any new episode of chest pain while the patient remained under observation and before discharge. Troponin T was measured at admission (i.e., within $6 \mathrm{~h}$ of chest pain) and at $12 \mathrm{~h}$ after the onset of chest pain. After ruling out an acute coronary syndrome by a negative serial cardiac troponin $\mathrm{T}$ (defined as peak value $0.03 \mu \mathrm{g} / \mathrm{L}$ at 6 and $12 \mathrm{~h}$ after the onset of symptoms) and by serial ECGs, the remaining patients underwent a twodimensional echocardiography. In a post-hoc analysis, all enrolled patients received a clinical score using the Global Registry of Acute Coronary Events (GRACE) [7] and Thrombolysis In Myocardial Infarction (TIMI) for NSTEMI/unstable angina [10]. The full details of the design and methods of the GRACE and TIMI risk scores have been previously published (available at www.outcomes-umassmed. org/grace and www.timi.org, respectively).

\section{Dobutamine stress echocardiography or echocardiogram}

The DSE was performed according to the current guidelines [5]: dobutamine was infused intravenously based on 3 -minute stages of 10, 20, 30, and $40 \mu \mathrm{g} / \mathrm{kg} / \mathrm{min}$. Atropine ( 0.25 to $1 \mathrm{mg}$ intravenously) was given if $85 \%$ of the agepredicted maximal heart rate was not reached (i.e., target heart rate) or if other endpoints were not reached at peak dobutamine dose. Endpoints included severe and/or extensive new wall motion abnormality, significant tachyarrhythmias, significant changes on the ECG, severe chest pain, significant increase or decrease in blood pressure $(>240 / 120 \mathrm{mmHg}$ or reduction of systolic pressure $>40 \mathrm{mmHg}$ ), or any intolerable side effect. A beta-blocker was administered intravenously to reverse the side effects, if they did not revert spontaneously and quickly after stopping the dobutamine infusion. For purpose of analysis, the left ventricular wall was divided into 16 segments and scored using a four-point scale (1, normal; 2, hypokinetic; 3, akinetic; and 4, dyskinetic) according to current guidelines [17]. A positive DSE was defined as the occurrence of new wall motion abnormality in at least one segment and was indicative for myocardial ischaemia. Myocardial ischaemia was not considered if akinetic segments at rest became dyskinetic during stress [18]. The images of the DSE were compared off-line side by side in quad screen format by two experienced investigators without knowledge of the patients' clinical data. In case of disagreement, a majority decision was achieved by a third investigator (JHC, OK, RB). The results were dichotomised as either positive or negative for ischaemia.

\section{Myocardial perfusion scintigraphy}

Myocardial perfusion scintigraphy (MPS) was performed in an outpatient setting at the physician's discretion, according 
to the guidelines of the American Society of Nuclear Cardiology [19] using a 2-day stress/rest protocol. A dose of $500 \mathrm{MBq}$ of technetium-99 m-tetrofosmin was administered at rest and at peak exercise. All patients were stressed with a bicycle ergometer with a starting workload of $50 \mathrm{~W}$, increasing by $25 \mathrm{~W}$ every $2 \mathrm{~min}$. Endpoints for exercise included achievement of $\geq 85 \%$ of age-predicted heart rate, recognisable chest pain, and $>2$-mm ST-segment depression [20]. All patients fasted both days, and antianginal medication was discontinued before the exercise test and restarted after exercise. Gated myocardial single-photon emission computed tomography was performed with the patient in a prone position.

Stress and rest perfusion images were scored in consensus by two experienced nuclear medicine physicians (HJV and FMvdZ) using a five-point semi-quantitative score for each of 17 myocardial segments. Perfusion defect severity was classified as normal (0), equivocally abnormal (1), mildly abnormal (2), moderately abnormal (3), or severely abnormal (4). Subsequently, summed stress score, summed rest score (SRS), and the difference between those scores (summed difference score, SDS) were calculated. $\mathrm{SDS} \geq 3$ was considered to indicate myocardial ischaemia. A fixed defect was defined as a SRS score of $\geq 3$. Mixed defects, partly reversible ( $\mathrm{SDS} \geq 3$ ) and partly fixed, were analysed as reversible. The results were dichotomised as either positive or negative for ischaemia and either as normal (no perfusion abnormalities) or abnormal (combined ischaemia and fixed defects). Assessment was performed blinded to patient data.

\section{Analysis of troponin T}

Troponin $\mathrm{T}$ levels were determined with an enzyme-linked immunosorbent assay method on an Elecsys 300 analyser (Roche Diagnostics, Almere, the Netherlands) using a thirdgeneration assay. A positive result was defined as a level $\geq 0.03 \mu \mathrm{g} / \mathrm{L}$, on admission or at $12 \mathrm{~h}$ after onset of symptoms.

\section{Follow-up}

Endpoint of the study was cardiovascular mortality, defined as myocardial infarction, life-threatening arrhythmia, or pulmonary oedema (based on clinical assessment, cardiac isoenzymes, ECG, or autopsy), or cerebrovascular accident. Unexpected death without an identified non-cardiac cause was also classified as cardiovascular death. Information on mortality was obtained from the National Registry on Mortality. For cause of death, the patients' general practitioners were contacted, and letters from hospital files were obtained, if necessary. Follow-up was complete for $96 \%$
(95\% for rule-out ACS patients and $98 \%$ for ACS patients). Median follow-up was 9.4 years (8.9-10.0 years) [9.4 years (8.9-10.0 years) for rule-out ACS patients and 9.2 years (8.9-9.9 years) for ACS patients].

\section{Statistical analysis}

Continuous variables were expressed as mean $\pm \mathrm{SD}$ when normally distributed, and median (interquartile range) if otherwise, and were compared using student's $T$-test and Mann-Whitney- $U$ test, respectively. Categorical variables were presented as frequencies, and compared using the chi-square test. Clinical variables were dichotomised with prospectively defined, clinically relevant cut-off points to improve readability of the data. Univariate and multivariate backward conditional Cox proportional hazard model was used to evaluate the associations between the outcome measures (primary endpoint: time to death). The variables incorporated into the GRACE and TIMI risk scores were excluded from the multivariate analysis. To estimate the discriminative value of Grace and TIMI risk scores, we calculated the C-statistic (area under the receiver operating characteristic curve). Survival analysis was performed with the Kaplan-Meier method and the log-rank test was used for comparisons of curves. The SPSS statistical package (SPSS PASW 18.0 for Windows, SPSS Inc., Chicago, Illinois) was used for analysis.

\section{Results}

The study comprised a total of 557 patients who gave informed consent. Thirty-three patients had serious noncardiac or cardiac diagnoses other than ACS. In the remaining 524 patients, 145 were diagnosed with ACS (positive serial troponin $\mathrm{T} \geq 0.03 \mu \mathrm{g} / \mathrm{L}$, recurrent angina and/or dynamic ECG changes during the observation period). These patients were defined as high-risk patients. The remaining 379 patients with ACS ruled out were discharged early, i.e. after the observation period. In these rule-out ACS patients, DSE was performed. Of these, 350 had an interpretable DSE. MPS was performed in 52 patients.

Table 1 shows the baseline characteristics of the entire cohort of chest pain patients presenting at the emergency room with a normal or non-diagnostic ECG. ACS patients were older and more often had a history of coronary artery disease. The use of beta-blockers and calcium antagonists was more prevalent in ACS patients whereas rule-out ACS patients more often had a normal ECG. DSE was positive in 23 rule-out ACS patients $(7 \%$ of 350 interpretable DSE). MPS was abnormal in 
Table 1 Baseline characteristics according to stratum

\footnotetext{
${ }^{\text {a }}$ Documented CAD defined as prior acute myocardial infarction, revascularisation, or documented coronary artery stenosis $\geq 50 \%$ ) on coronary angiogram. $A C E=$ angiotensin-converting enzyme; $A C S=$ acute coronary syndrome; $C A D=$ coronary artery disease, $C V A=$ cerebrovascular accident; $D S E$ dobutamine stress echocardiography $=; M P S=$ Myocardial perfusion scintigraphy, $T I A=$ transient ischaemic attack
}

\begin{tabular}{|c|c|c|c|}
\hline Variables & Rule-out ACS $(n=379)$ & $\operatorname{ACS}(n=145)$ & $\mathrm{p}$ \\
\hline Age (years) mean $\pm \mathrm{SD}$ & $56 \pm 12$ & $62 \pm 11$ & $<0.001$ \\
\hline Men & $220(58)$ & $97(67)$ & 0.064 \\
\hline Diabetes mellitus & $36(10)$ & $20(14)$ & 0.155 \\
\hline Hypertension & $145(38)$ & $61(42)$ & 0.424 \\
\hline Current cigarette smoking & $141(37)$ & $44(30)$ & 0.124 \\
\hline Hypercholesterolaemia & $133(35)$ & $50(35)$ & 0.896 \\
\hline Family history of CAD & $193(51)$ & $66(46)$ & 0.268 \\
\hline Documented CAD ${ }^{\mathrm{a}}$ & $80(21)$ & $43(30)$ & 0.039 \\
\hline Previous myocardial infarction & $59(16)$ & $26(18)$ & 0.511 \\
\hline History of chronic heart failure & $2(1)$ & $3(2)$ & 0.104 \\
\hline Peripheral artery disease & $50(13)$ & $20(14)$ & 0.835 \\
\hline TIA/CVA & $22(6)$ & $9(6)$ & 0.861 \\
\hline \multicolumn{4}{|l|}{ Medication on admission } \\
\hline Aspirin & $123(33)$ & $59(41)$ & 0.076 \\
\hline Beta-blocker & $104(27)$ & $59(41)$ & 0.003 \\
\hline Calcium antagonist & $56(15)$ & $35(24)$ & 0.011 \\
\hline ACE inhibitor & $43(11)$ & $20(14)$ & 0.441 \\
\hline Statin & $65(17)$ & $27(19)$ & 0.692 \\
\hline \multicolumn{4}{|l|}{ Baseline ECG category } \\
\hline Normal & $228(60)$ & $56(39)$ & $<0.001$ \\
\hline \multicolumn{4}{|l|}{ Cardiac imaging results } \\
\hline Positive DES & $23 / 350(7 \%)$ & & \\
\hline Abnormal MPS & $8 / 52(15 \%)$ & & \\
\hline Reversible perfusion defects on MPS & $4 / 52(7 \%)$ & & \\
\hline GRACE risk score (25th, 75 th percentiles) & $75(57-96)$ & $89(74-107)$ & $<0.001$ \\
\hline TIMI risk score (25th, 75th percentiles) & $1(0-2)$ & $2(1-3)$ & $<0.001$ \\
\hline
\end{tabular}

8 patients (15\% of 52 MPS performed), and showed myocardial ischaemia in 4 patients (7\% of 52 MPS performed). The GRACE risk score was low in both groups (75 (57-96) in rule-out ACS vs. 75 (57-96) in ACS patients, as well as TIMI risk scores (1 (0-2) vs. 2 (1-3) (median, (25th, 75th percentiles)), respectively. Both GRACE and TIMI risk scores were significantly higher in ACS patients.
During follow-up, death occurred in 78 patients (15\%), $43(11 \%)$ in the rule-out ACS group and $35(24 \%)$ in the ACS group $(p<0.001)$. Causes of death are displayed in Table 2. Of all deceased patients, $45(58 \%)$ had a cardiovascular cause of death. In the rule-out ACS group, 21 patients (49\% of deaths in this group) died of a cardiovascular cause compared with $24(69 \%)$ in the ACS group $(p<0.001)$.
Table 2 Cause of death according to risk group

$A C S=$ acute coronary syndrome; $C V A=$ cerebrovascular accident

\begin{tabular}{lllll}
\hline & $\begin{array}{l}\text { Overall } \\
(n=524)\end{array}$ & $\begin{array}{l}\text { Rule-out ACS } \\
(n=379)\end{array}$ & $\begin{array}{l}\text { ACS } \\
(n=145)\end{array}$ & $p$ \\
\hline Follow-up & $9.4(8.9-10.0)$ & $9.4(8.9-10.0)$ & $9.2(8.9-9.9)$ & \\
Death (all-cause) & $78(15 \%)$ & $43(11 \%)$ & $35(24 \%)$ & $<0.001$ \\
Time-to-death & $6.1(2.5-7.6)$ & $6.1(2.5-7.7)$ & $6.0(2.5-7.6)$ & \\
Cardiovascular death & $45(58 \%)$ & $21(49 \%)$ & $24(69 \%)$ & $<0.001$ \\
- Sudden unexpected death & 10 & 3 & 7 & \\
- Acute myocardial infarction & 6 & 5 & 4 & \\
- Heart failure & 5 & 1 & 2 & \\
- CVA & 3 & 1 & 1 & \\
- Acute abdominal aortic aneurysm & 1 & 10 & 10 & \\
- Unknown & 20 & & 1 & \\
\hline
\end{tabular}


Table 3 Univariate and multivariate Cox regression analysis for cardiovascular mortality of clinical parameters and cardiac imaging in rule-out ACS patients

\footnotetext{
${ }^{a}$ Documented CAD defined as prior acute myocardial infarction, revascularisation, or documented coronary artery stenosis $\geq 50 \%$ ) on coronary angiogram. $A C E=$ angiotensin-converting enzyme; $A C S=$ acute coronary syndrome; $C A D=$ coronary artery disease, $C V A=$ cerebrovascular accident; $D S E=$ dobutamine stress echocardiography; $M P S=$ Myocardial perfusion scintigraphy, $T I A=$ transient ischaemic attack

b according to GRACE risk score categorisation of patients for non-ST-segment elevation ACS: low (1-108), intermediate (109-140), and high (141-372) risk

c according to TIMI risk score categorisation for unstable angina/NSTEMI, low (0-2), intermediate (3-4), and high (5-7) risk
}

Fig. 1 Survival of rule-out ACS chest pain patients with a normal or non-diagnostic ECG.

Kaplan-Meier plots are displayed showing survival stratified by GRACE risk score categorisation of patients for non-ST-segment elevation ACS: low (1-108), intermediate (109$140)$, and high (141-372) risk

\begin{tabular}{|c|c|c|c|c|}
\hline \multirow[t]{2}{*}{ Variables } & \multicolumn{2}{|l|}{ Univariate analysis } & \multicolumn{2}{|l|}{ Multivariate analysis } \\
\hline & Point estimate & $\mathrm{p}$ & Point estimate & $\mathrm{P}$ \\
\hline Age $>65$ years & $14.61(4.91-43.45)$ & $<0.001$ & & \\
\hline Men & $0.65(0.28-1.55)$ & 0.656 & & \\
\hline Diabetes mellitus & $2.55(0.86-7.58)$ & 0.092 & & \\
\hline Hypertension & $1.50(0.64-3.53)$ & 0.356 & & \\
\hline Current cigarette smoking & $1.34(0.85-2.11)$ & 0.203 & & \\
\hline Hypercholesterolaemia & $0.91(0.370-2.26)$ & 0.844 & & \\
\hline Family history of CAD & $0.92(0.39-2.15)$ & 0.838 & & \\
\hline Documented CAD ${ }^{\mathrm{a}}$ & $5.24(2.21-12.44)$ & $<0.001$ & & \\
\hline Previous myocardial infarction & $5.22(2.21-12.29)$ & $<0.001$ & & \\
\hline Known chronic heart failure & $14.63(1.96-109.43)$ & 0.009 & $10.41(1.17-92.37)$ & 0.035 \\
\hline Peripheral artery disease & $1.68(0.56-4.99)$ & 0.352 & & \\
\hline TIA/CVA & $4.14(1.39-12.32)$ & 0.011 & & \\
\hline Aspirin use & $3.60(1.49-8.67)$ & 0.004 & & \\
\hline Beta-blocker use & $1.34(0.54-3.32)$ & 0.529 & & \\
\hline Calcium antagonist use & $4.55(1.92-10.80)$ & 4.547 & & \\
\hline ACE inhibitor use & $4.50(1.82-11.16)$ & 0.001 & $4.38(1.70-11.29)$ & 0.002 \\
\hline Statin use & $0.23(0.03-1.68)$ & 0.146 & & \\
\hline Normal baseline ECG & $0.15(0.05-0.44)$ & 0.001 & & \\
\hline \multicolumn{5}{|l|}{ Cardiac imaging results } \\
\hline Positive DSE & $0.64(0.09-4.75)$ & 0.660 & & \\
\hline Abnormal MPS & - & - & & \\
\hline Reversible perfusion defects on MPS & - & - & & \\
\hline GRACE risk score $^{\mathrm{b}}$ & $6.82(3.88-11.99)$ & $<0.001$ & $7.41(4.05-13.57)$ & $<0.001$ \\
\hline TIMI risk score $^{\mathrm{c}}$ & $4.88(2.07-11.49)$ & $<0.001$ & & \\
\hline
\end{tabular}


The GRACE and TIMI risk scores demonstrated a very good discrimination for long-term cardiovascular mortality. For rule-out ACS patients, C-statistics were 0.829 and 0.803 , and for ACS patient C-statistics were 0.807 and 0.715 for the GRACE and TIMI scores, respectively.

Table 3 shows the results of the univariate and multivariate Cox regression analysis for cardiovascular mortality of clinical parameters and cardiac imaging. An age above 65 years, documented coronary artery disease, a previous myocardial infarction, history of chronic heart failure, transient ischaemic attack or cerebrovascular accident, aspirin and ACE inhibitor use, an abnormal baseline electrocardiogram as well as GRACE and TIMI score were predictors of cardiovascular mortality. None of the patients with an MPS suggestive of myocardial ischaemia $(n=4)$ or abnormal MPS $(n=8)$ had cardiovascular mortality during follow-up. A positive DSE was not identified as a prognostic marker for cardiovascular mortality. In multivariate analysis, known chronic heart failure, ACE inhibitor use, and GRACE score were independent predictors of cardiovascular mortality. Figure 1 shows the Kaplan Meier curves stratified by GRACE risk score categorisation of patients for non-STsegment elevation ACS. In patients with intermediate (109 to 140) and high (141 to 372) GRACE risk scores, cardiovascular mortality ( $75 \%$ and $23 \%$ respectively) was notably higher than in the low GRACE risk scores (1 to $108)$ rule-out ACS patients $(2 \%)$.

\section{Discussion}

In our rule-out ACS population of chest pain patients with a normal or non-diagnostic electrocardiogram, long-term cardiovascular mortality during a 9-year follow-up was low $(6 \%)$, and comparable to previous studies on chest pain patients without ST elevation on ECG [7-9]. The long-term cardiovascular prognosis of patients with a positive DSE or reversible or irreversible defects on MPS was as good as the prognosis of patients with normal DSE or MPS. However, GRACE and TIMI risk scores had predictive values with $\mathrm{C}$-statistics comparable to the values found in the ACS populations for which they were designed [21, 22], but this is the first study, to our knowledge, to show a long-term value for these scoring systems in a rule-out ACS population.

Previous studies have shown a predictive value of DSE for cardiac adverse events [11, 12]. However, these studies involved single-centre experiences in very heterogeneous populations of consecutive patients undergoing DSE. Moreover, these studies did not evaluate cardiovascular mortality as a separate event. In another study by Steinberg [13], DSE was predictive for cardiovascular mortality, but the population consisted of patients referred for coronary angiography with mortality rates twice as high as in our study. In an emergency department setting of patients with suspected myocardial infarction, MPS was predictive for cardiac mortality, but again the cardiac mortality rate was more than twice as high as the cardiovascular mortality in our study [23]. The absence of a predictive value for DSE and MPS in our study might thus be related to the selection of patients with an already apparent low risk of cardiovascular mortality.

There are several limitations to our study. First, we only studied cardiovascular mortality with no secondary endpoints such as myocardial infarction and revascularisation. In a previous study, we have shown the strong and independent short-term predictive value of DSE for these endpoints in our populations [24]. However, for long-term risk stratification, mortality endpoints are more relevant as shown by a continuation of the survival curve [13], in contrast to converging and flattening curves of combined endpoints $[11,15]$.

Second, MPS was only performed in a very small subset of the population. Nonetheless, with an equally low cardiovascular mortality rate ( $4 \%$, data not shown), this subset is comparable to the entire rule-out ACS cohort.

In conclusion, imaging for inducible myocardial ischaemia by DSE and MPS does not predict long-term cardiovascular mortality in chest pain patients with a normal or non-diagnostic electrocardiogram after ruling out ACS. The TIMI and GRACE scores, however, are accurate predictors in these patients with low long-term cardiovascular mortality.

Competing interests None.

Ethics approval Approval from the ethics committees at each of the recruiting sites was obtained and patients' consents were received.

Funding for this work was provided by the Netherlands Heart Foundation (grant NHS 96.172).

Open Access This article is distributed under the terms of the Creative Commons Attribution Noncommercial License which permits any noncommercial use, distribution, and reproduction in any medium, provided the original author(s) and source are credited.

\section{References}

1. Bassand J, Hamm CW, Ardissino D, et al. Guidelines for the diagnosis and treatment of non-ST-segment elevation acute coronary syndromes. Eur Heart J. 2007;28:1598-660.

2. Anderson JL, Adams CD, Antman EM, et al. ACC/AHA 2007 guidelines for the management of patients with unstable angina/ 
non ST-elevation myocardial infarction: a report of the American College of Cardiology/American Heart Association Task Force on Practice Guidelines (Writing Committee to Revise the 2002 Guidelines for the Management of Patients With Unstable Angina/Non ST-Elevation Myocardial Infarction): developed in collaboration with the American College of Emergency Physicians, the Society for Cardiovascular Angiography and Interventions, and the Society of Thoracic Surgeons: endorsed by the American Association of Cardiovascular and Pulmonary Rehabilitation and the Society for Academic Emergency Medicine. Circulation. 2007;116:e148-304.

3. Westerhout $\mathrm{CM}, \mathrm{Fu}$ Y, Lauer MS, et al. Short- and long-term risk stratification in acute coronary syndromes: the added value of quantitative ST-segment depression and multiple biomarkers. J Am Coll Cardiol. 2006;48:939-47.

4. Sanchis J, Bodí V, Núñez J, et al. Usefulness of early exercise testing and clinical risk score for prognostic evaluation in chest pain units without preexisting evidence of myocardial ischemia. Am J Cardiol. 2006;97:633-5.

5. Rodgers GP, Ayanian JZ, Balady G, et al. American College of Cardiology/American Heart Association Clinical Competence statement on stress testing: a report of the American College of Cardiology/American Heart Association/American College of Physicians-American Society of Internal Medicine Task Force on Clinical Competenc. J Am Coll Cardiol. 2000;36:1441-53.

6. Stein RA, Chaitman BR, Balady GJ, et al. Safety and utility of exercise testing in emergency room chest pain centers: an advisory from the Committee on Exercise, Rehabilitation, and Prevention, Council on Clinical Cardiology, American Heart Association. Circulation. 2000;102:1463-7.

7. Eagle KA, Lim MJ, Dabbous OH, et al. A validated prediction model for all forms of acute coronary syndrome: estimating the risk of 6-month postdischarge death in an international registry. JAMA. 2004;291:2727-33.

8. Granger CB, Goldberg RJ, Dabbous $\mathrm{O}$, et al. Predictors of hospital mortality in the global registry of acute coronary events. Arch Intern Med. 2003;163:2345-53.

9. Boersma E, Pieper KS, Steyerberg EW, et al. Predictors of outcome in patients with acute coronary syndromes without persistent ST-segment elevation. Results from an international trial of 9461 patients. The PURSUIT Investigators. Circulation. 2000;101:2557-67.

10. Antman EM, Cohen M, Bernink PJ, et al. The TIMI risk score for unstable angina/non-ST elevation MI: A method for prognostication and therapeutic decision making. JAMA. 2000;284:835-42.

11. Biagini E, Elhendy A, Bax JJ, et al. Seven-year follow-up after dobutamine stress echocardiography: impact of gender on prognosis. J Am Coll Cardiol. 2005;45:93-7.

12. Chuah SC, Pellikka PA, Roger VL, et al. Role of dobutamine stress echocardiography in predicting outcome in 860 patients with known or suspected coronary artery disease. Circulation. 1998;97:1474-80.

13. Steinberg EH, Madmon L, Patel CP, et al. Long-term prognostic significance of dobutamine echocardiography in patients with suspected coronary artery disease: results of a 5-year follow-up study. J Am Coll Cardiol. 1997;29:969-73.

14. Travin MI, Heller GV, Johnson LL, et al. The prognostic value of ECG-gated SPECT imaging in patients undergoing stress Tc-99 m sestamibi myocardial perfusion imaging. J Nucl Cardiol. 2000;11:253-62.

15. Schinkel AFL, Elhendy A, Van Domburg RT, et al. Long-term prognostic value of dobutamine stress $99 \mathrm{mTc}$-sestamibi SPECT: single-center experience with 8-year follow-up. Radiology. 2002;225:701-6.

16. Bholasingh R, Cornel JH, Kamp O, et al. The prognostic value of markers of inflammation in patients with troponin T-negative chest pain before discharge from the emergency department. Am J Med. 2003;115:521-8.

17. Schiller NB, Shah PM, Crawford M, et al. Recommendations for quantitation of the left ventricle by two-dimensional echocardiography. American society of echocardiography committee on standards, subcommittee on quantitation of two-dimensional echocardiograms. J Am Soc Echocardiogr. 1989;2:358-67.

18. Arnese M, Fioretti PM, Cornel JH, et al. Akinesis becoming dyskinesis during high-dose dobutamine stress echocardiography: a marker of myocardial ischemia or a mechanical phenomenon? Am J Cardiol. 1994;73:896-9.

19. (2001) Updated imaging guidelines for nuclear cardiology procedures, part 1. J Nucl Cardiol 8: G5-G58

20. Gibbons RJ, Balady GJ, Bricker JT, et al. ACC/AHA 2002 guideline update for exercise testing: summary article. A report of the American College of Cardiology/American Heart Association Task Force on Practice Guidelines (Committee to Update the 1997 Exercise Testing Guidelines). J Am Coll Cardiol. 2002;40:1531-40.

21. Yan AT, Jong P, Yan RT, et al. Clinical trial-derived risk model may not generalize to real-world patients with acute coronary syndrome. Am Heart J. 2004;148:1020-7.

22. Morrow DA, Antman EM, Charlesworth A, et al. TIMI risk score for ST-elevation myocardial infarction: a convenient, bedside, clinical score for risk assessment at presentation: an intravenous nPA for treatment of infarcting myocardium early II trial substudy. Circulation. 2000;102:2031-7.

23. Launbjerg J, Fruergaard P, Jacobsen HL, et al. The long-term predictive value of an exercise thallium-201 scintigraphy for patients with acute chest pain but without myocardial infarction. Coron Artery Dis. 1993;4:195-200.

24. Bholasingh R, Cornel JH, Kamp O, et al. Prognostic value of predischarge dobutamine stress echocardiography in chest pain patients with a negative cardiac troponin T. J Am Coll Cardiol. 2003;41:596-602. 